\title{
EMERGENT PROCESSES
}

\author{
ADRIAN LAHOUD \\ Faculty of Design, Architecture and Building \\ University of Technology, Sydney \\ PO Box 123, Broadway, NSW 2007, Australia
}

Adrian.lahoud@uts.edu.au

\begin{abstract}
Emergence is a well established though highly contested concept in science that is found in a range of disciplines from biology and artificial life to physics and complexity. It is not in doubt that emergence is of interest to other fields including design, though the manner of its relation to this discipline is much less clear and its effect remains an open question.

This paper will argue that emergence has set in motion a shift in the way we understand design. Building on the theoretical and philosophical resources provided by Gilles Deleuze (1994) and Manuel Delanda (2002) in particular, emergence marks a reorientation in the techniques and practices of designing, away from the specific designation of an objects qualities, towards the specific designation of an environment in which the object's qualities might emerge from a range of potentialities.
\end{abstract}

\section{Introduction}

Emergence is a well established though highly contested concept in science that is found in a range of disciplines from biology and artificial life to physics and complexity. It is not in doubt that emergence is of interest to other fields including design, though the manner of its relation to this discipline is much less clear and its effect remains an open question. Architecture or urbanism, for example, would place quite different demands on a concept like emergence than those placed on it by neuroscience. Whilst the former might engage with those aspects of emergence that pertain to the generation of form or pattern as it relates to a city; the later might try to explain the development of cognition as it relates to a substrate of neural networks. Silberstein and McGeever define emergence as follows:

"...features of systems or wholes that possess causal capacities not reducible to any of the intrinsic causal capacities of the parts nor to any of the (reducible) relations between the parts. Emergent properties are properties of a system taken as a whole which exert a causal influence on the parts of the system consistent with, but distinct from, the causal capacities of the parts themselves." (182)

This paper will argue that emergence has set in motion a shift in the way we understand design. Building on the theoretical and philosophical resources provided by Gilles Deleuze (1994) and Manuel Delanda (2002) in particular, emergence marks a reorientation in the techniques and practices of designing, away from the specific designation of an objects qualities, towards the specific designation of an environment in which the object's qualities might emerge from a 
range of potentialities. These environments are simply mathematical spaces whose properties we can both describe and attach meaning to. Though fields like biology (Strogatz 1994) or economics (Krugman 1996) might use these tools as analytical devices to gain information about complex phenomena (like the tendency for business agglomerations to form), in design the question of empirical use value takes on a different inflection since evaluation criteria are rarely self evident and not always quantifiable.

With these qualifications regarding empirical status in mind, for designers emergence still marks a fundamental movement away from the direct production of form - what is being reconceptualised here is the mode of control over the designed object. Rather than manipulate the materiality of the object or the object's representation directly, an emergent designer manipulates the properties of a mathematical space in which an infinite series of potential forms might be packed. ${ }^{1}$ The emergence or actualisation of a specific form out of this space is understood to be a contingent product of the self-organized interaction of the space's different components. The danger here is that emergence comes to be understood as a naturalised model of formal development, authenticated via the intimate connection between the object and the world from which it emerges. ${ }^{2}$

The shift away from the direct and directed production of the architectural artefact towards an engagement with the space in which the artefact exists as potential carries with it all the predictable revaluations of authorship that attend to many new mediums.

In this instance however, what is new is the way questions regarding the status of control, continuity and difference can be posed.

To claim that the production of some object is the natural consequence of a space or environmental field as represented digitally or mathematically, is to make a claim for a certain continuity between the environment and the objects that arise out of it. Emergence, crucially, would describe a mode in which this continuity is not simply the elaboration of that which already implicitly exists in the environment as a possibility, but the very creativity of the environment itself, its ability to self-generate pattern and form.

In order to substantiate this claim for material creativity we would need to make an assessment of the qualities of the object produced with regards to the environment and decide whether this represented a novel development that could not be retrospectively explained by its initial state. In other words, because emergence is an account of the development of form, we would have to understand the history of the world or system that conspires to produce said form and decide whether the material sequence of this history is evidence of novelty or simple mechanical elaboration. Whilst a mechanical elaboration would be evidence of continuity, it would not be evidence of emergence because it would be reducible to the sum of the component interactions. Irreducibility is seen as key feature of 'strong' or 'ontological' forms of emergence, in that the behaviour of the system taken in total cannot be reduced to the sum of its component interactions. As Silberstein and McGeever note "(Ontological) ...eemergence therefore entails the failure of part-whole reductionism." (182)

Further, simple mechanical interaction is invariant with regards to time: time invariance or reversibility must follow from a deterministic and mechanical view of the world. If given a sequence of events, time plays no part in determining the properties of that sequence, then we can assume that given perfect knowledge with respect to any stage of the sequence we can clearly determine the properties of the sequence at other stages.

This conception of time as nothing but a neutral container for the clockwork elaboration of a predetermined sequence has been critiqued in philosophy through the work of Henri Bergson (1959) and in science through the work of llya Prigogine (1996). Emergence on the other hand implies a conceptualisation of time that is irreducible to metric determination and more importantly works to drive differentiation.

\section{Epistemological emergence}

To explain this further it is worth introducing a distinction between the properly emergent and the epistemologically emergent (though this in itself will require further elaboration). The epistemologically emergent is that which can be explained by recourse to the history of the system, but that with regards to an observer is unanticipated.

In other words the historical account of an object's development, its morphogenesis, can be given through a description of the mechanical interaction of its components. Though the complexity of these interactions might mean that predictability is difficult, it does not mean that 
predictability is impossible, only that we have insufficient information to describe the system correctly. The difference between that which is difficult to predict because of complexity and that which is impossible to predict is crucial with regards to emergence. Whilst both might produce unexpected results with regards to an observer, only the latter would be evidence of novelty. This is because in the former case, it is the observational or descriptive apparatus that is blind with regards to the system, where as in the latter case, the system itself is blind with regards to its future. As Silberstein and McGeever note:

"A property of an object or system is epistemologically emergent if the property is reducible to or determined by the intrinsic properties of the ultimate constituents of the object or system, while at the same time it is very difficult for us to explain, predict or derive the property on the basis of the ultimate constituents. Epistemologically emergent properties are novel only at a level of description. For example, even systems with very few parts and with simple mathematical rules can sometimes be said to exhibit epistemological emergence." (186)

With regards to digital design processes we should be careful to distinguish between the unanticipated and the unaccountable. The power of computational systems can regularly produce unanticipated effects in design processes, but these effects will be accounted for through the simple mechanical interaction of the systems codified components.

\section{Strong and weak emergence}

The higher order development that has been crudely referred to as the properly emergent can also said to exist in a variety of types. In order to produce a more nuanced understanding of what constitutes emergence we should distinguish between its strong and weak forms, not simply to construct contrasting categories for a taxonomy of emergent behaviour but to see how these categories pose two very different horizons for design.

An example of weak emergence can be found in the use of analogue modelling techniques.

At the Institute for Lightweight structures in Stuttgart Germany, Frei Otto (Bach and Otto 1988) has developed form finding techniques using simple material analogues like soap film that recall Antoni Gaudi's inverted catenary model for the Sagrada Familia in Spain. Frei Otto's use of soap film for these experiments takes advantage of the material tendencies of the soap molecules. At a molecular level soap molecules will arrange themselves into configurations that minimise energy bonds between them, the emergent effect at a macro level is that the soap film will find a form that minimises its surface area, thus 'solving' the problem of spanning a surface efficiently.

There is a characteristic entanglement of scales at work here with energy minimisation at the level of the individual molecules driving a process structured by the surface tension of the components taken as a population- to produce an efficient configuration at the level of the total system. Though the shape of the surface might be changing in each case, the system is being focused on the same goal, namely optimisation. In fact the soap film will always move through a sequence of different volumes until it settles into its most efficient state. The time in which the soap film is 'finding' its equilibrium state can be thought of as a series of samples from a search space, in which each less efficient configuration is discarded for a more efficient one.

What is important to note here is this notion of a search space or more correctly a state space. In the case of the soap film, the state space of possible configurations that the soap film 'explores' is itself closed and stable. Its closure is less relevant, this simply means that the potential types of shape the soap can acquire are bounded, what is more important is that it is stable. In other words the system will always be driving towards a single outcome, and that once this outcome has been actualised, the systems behaviour becomes locked in place.

\section{State spaces and search algorithms}

The concept of state spaces can be explained more fully through an example taken from the world of computer animation. Inverse kinematics is a means of defining relationships between parts in a digital computer model such that each component will at its joint propagate a force to its adjoining component. It is used to efficiently model the skeletal system of characters in animation because it provides an efficient way to control joint rotation between rigid elements. It is efficient because inverse kinematics, unlike forward kinematics, propagates forces from the bottom of a hierarchy back up the chain of components (figure 1) (Elias). 


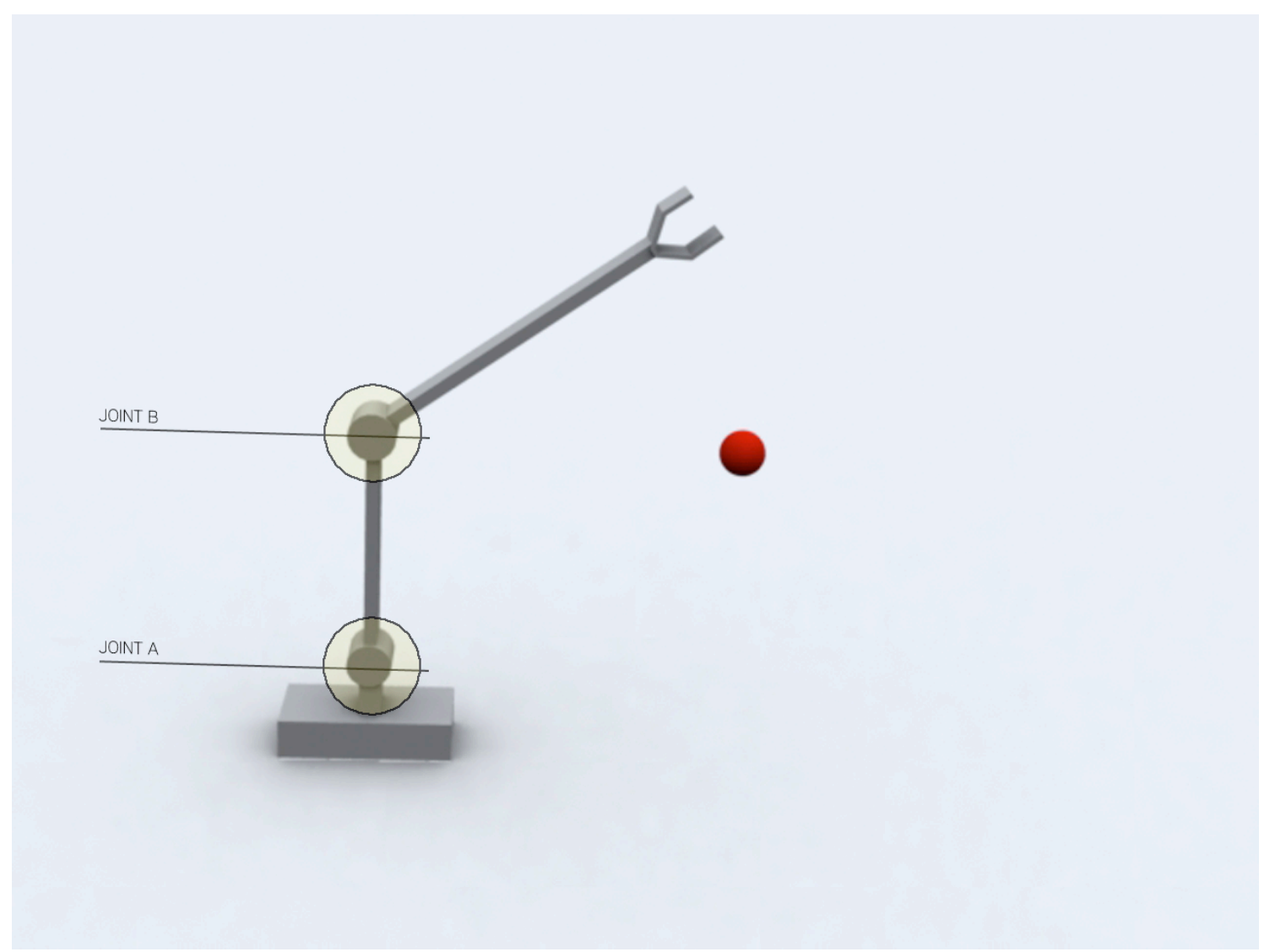

Figure 1: Robot arm 01

With regular or forward kinematics, to animate an arm picking up a ball we would first need to rotate the shoulder, extend the arm and open the palm before finally wrapping the fingers around the ball to clutch it. With inverse kinematics I define a series of properties for each joint, the shoulder is a ball and socket type joint, it can rotate within this range, the elbow can extend this far, fingers cannot bend backwards etc. Further each joint is given a weight or resistance, when we move naturally the hand opens early to anticipate grabbing the ball, the elbow moves quickly etc. Now once we have defined these relational properties between members, in inverse kinematics we simply grab the fingers and move them towards the target object, the other components automatically respond based on the properties of the joints in between.

The difficulty arises when we ask the computer generated arm to find the object, rather than simply direct it towards the object manually (figure 2), especially for systems in which joint rotation can move across three axes. Whilst the starting point and goal object or target points of the arm are known and we can solve the shortest possible route between these points very easily, what is much more difficult is the solution for what angles the joints will assume during this movement. In fact for circumstances with 3 or more joints there is often no analytical solution to the problem (figure 3). 


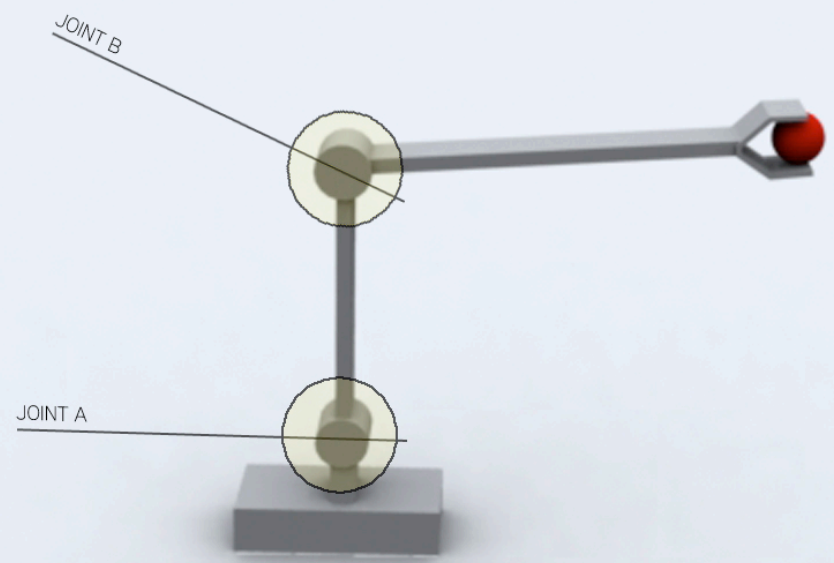

Figure 2: Robot arm 02

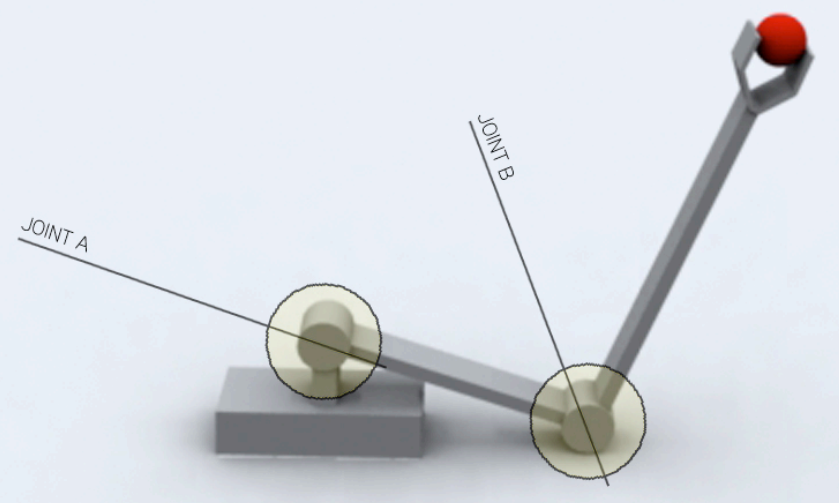

Figure 3: Robot arm 03 
Staying with this simple example, when we ask the computer to find the most efficient way, given the joint constraints, to find its object, one solution that is often used is to deploy an iterative algorithm (non-linear programming techniques are often the only ones that will be able to deliver any solutions to the problem). The iterative algorithm can be said to search the space of possible solutions from a given starting point, when more efficient values are returned they are favoured over less efficient ones. The space of possible solutions can be called a search space for the algorithm.

What does a search space look like? (figure 4) In the case of this robot arm, we could assign to each joint a variable measured in degrees. Plotting each of the rotational ranges of each joint on separate axis would allow us to describe a space bounded by the 180 degree limit of the maximum extension of each joint.

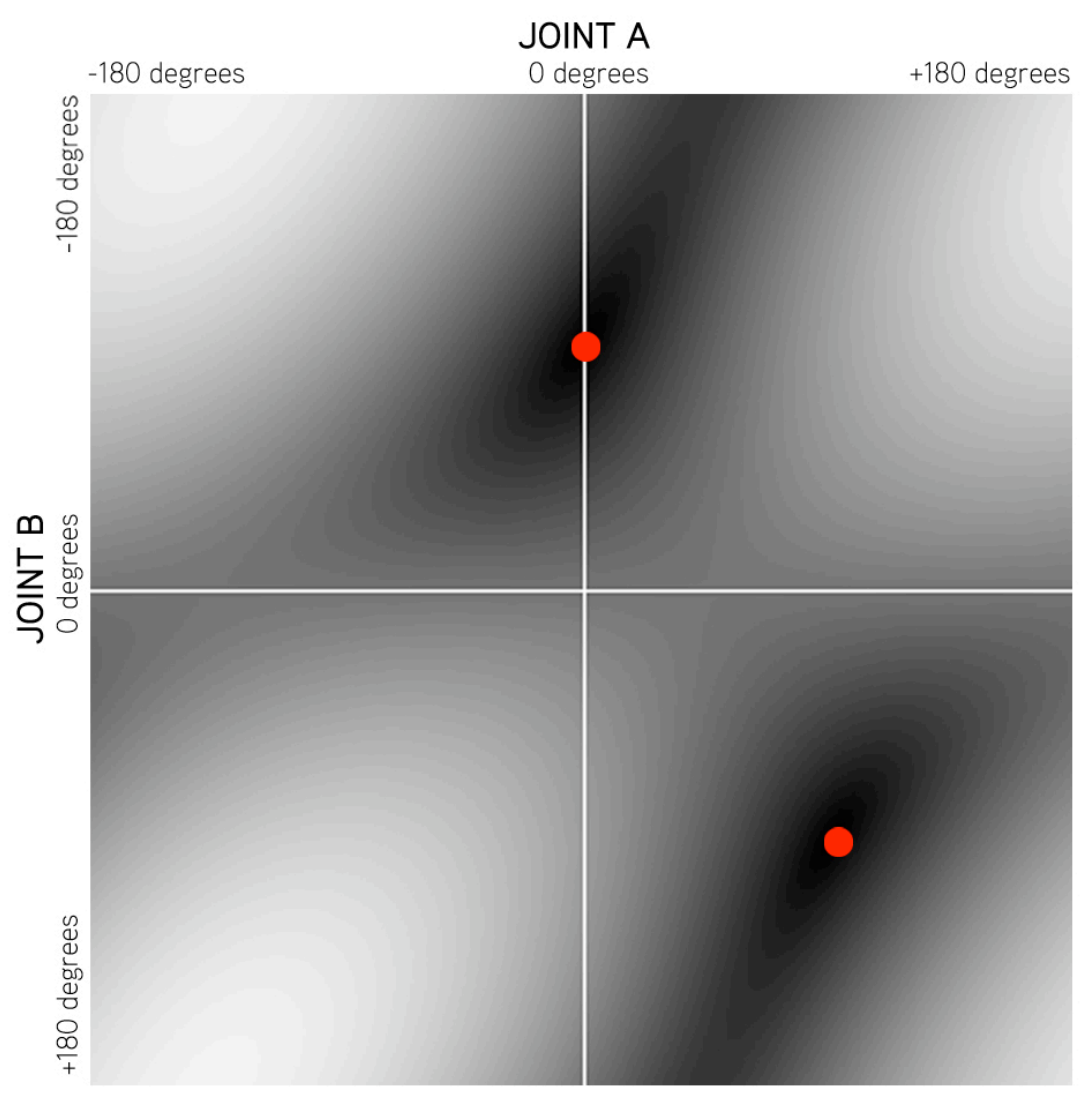

Figure 4: Graph

What is critical here is to understand here is that this graph is not a representation of the real two dimensional space in which the arm exists, we could not simply super-impose the arm onto this graph because the graph is measuring degrees and the physical space of the robot arm picture would be measured in distance units. What this graph is showing is all the potential configurations of joint angles in the arm. We can see that two points on the graph refer to the two combinations of angles on each joint that will get us to the target.

The darker areas show combinations of joint angles that get the hand closer to the target, the lighter areas show combinations of joint angles that move the arm further away from the target. This landscape describes all possible combinations of movement for the arm- it is a perfectly complete description. This space of possibility can be subdivided infinitely, there are literally countless ways of occupying this space, and yet it is an infinity that is delimited by the extent of the graph. More interestingly however we note that it is not an undifferentiated space, it is literally a landscape (figure 5). The algorithm is simply a machine that explores the landscape by looking for the minimum or darkest point using mathematical differentiation, similar to trying to find the maxima or minima on a curve. 


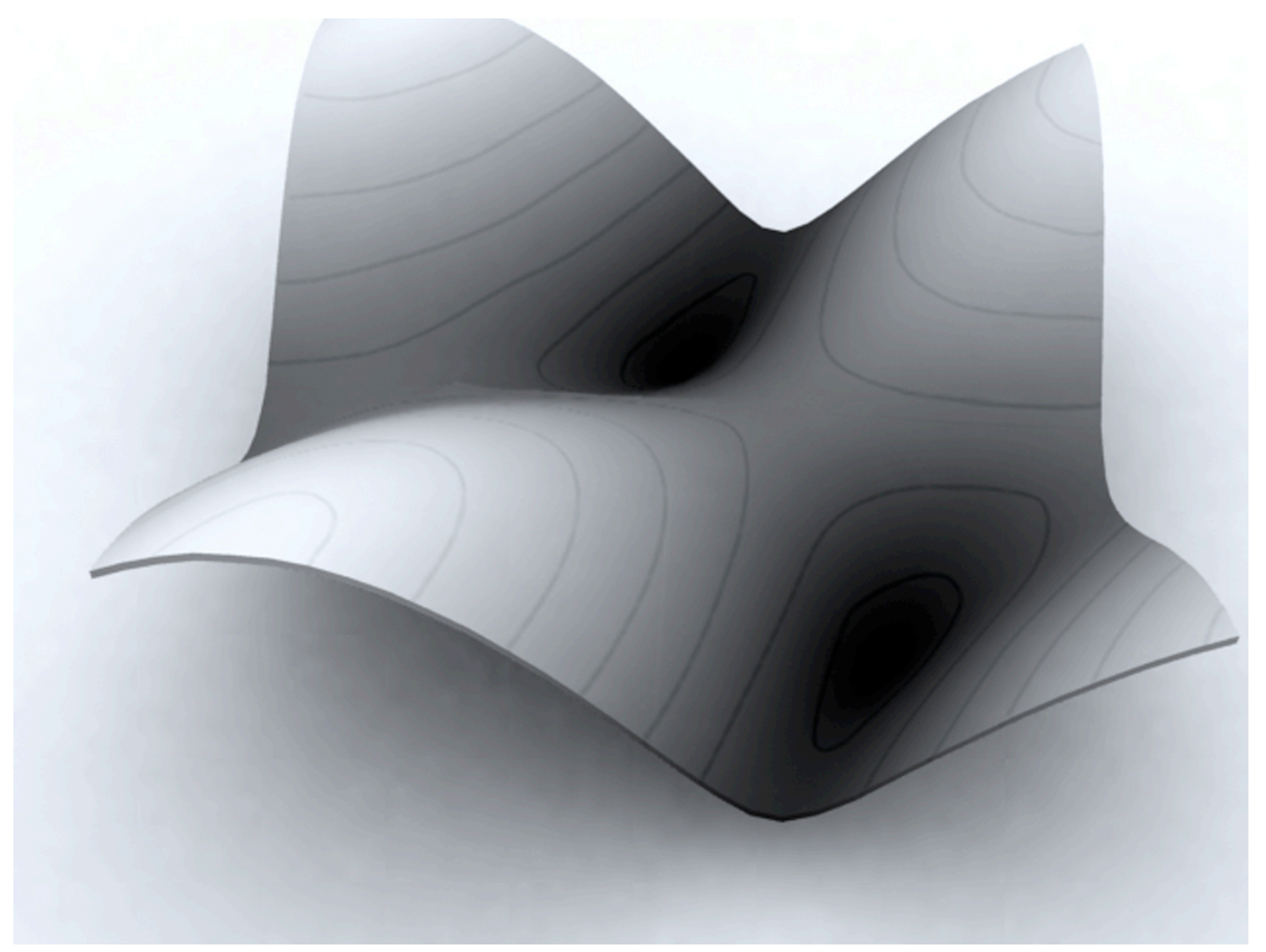

Figure 5: Graph landscape

We can treat any system in which we can describe the degrees of freedom of its parts in the same way. Conceptually, we might therefore treat the landscape as more than an expression of possibility but as the very field that determines the behaviour of the system (figure 6). Crudely speaking we could reverse the causal chain and see the properties of the graph or landscape driving the arm and not the other way around. This is how evolutionary or algorithmic design processes operate. Evolutionary design processes produce iterations of form by populating successive locations in these spaces. Formal variation in the object is driven by the variation of the mathematical landscape occupied at any one time. Considering the system at this level provides a description of the overall tendencies, potentials and most importantly impossibilities that govern specific behaviour. The specific history of a system's behaviour is given by the line a point inscribes as it is travelling across this mathematical surface.

\section{Basins of attraction and bifurcations}

The minima can be thought of as a basin of attraction (figure 7), so whenever the point tips over the precipice or edge of one of the higher contours it will drop into a different basin of attraction and thus change its behaviour. An attractor is a point that governs a specific pattern of behaviour (many attractors can exist in the same space). A basin of attraction is the zone of influence for the given attractor (when a point enters into a basin of attraction its behaviour is influenced by that attractor). A system poised at the border of two basins of attraction can be said to be at a bifurcation point (figure 8), with the slightest perturbation sending it into a different path. Stuart Kaufmann has suggested that complex systems can maximise their adaptability by locating themselves on these bifurcations zones, effectively increasing their freedom to change behaviour.

"Networks on the boundary between order and chaos may have the flexibility to adapt rapidly and successfully through the accumulation of useful variations. In such poised systems, most mutations have small consequences because of the systems' homeostatic nature. A few mutations, however, cause larger cascades of change. Poised systems will therefore typically adapt to a changing environment gradually, but if necessary, they can occasionally change rapidly." (Kaufmann 81 ) 


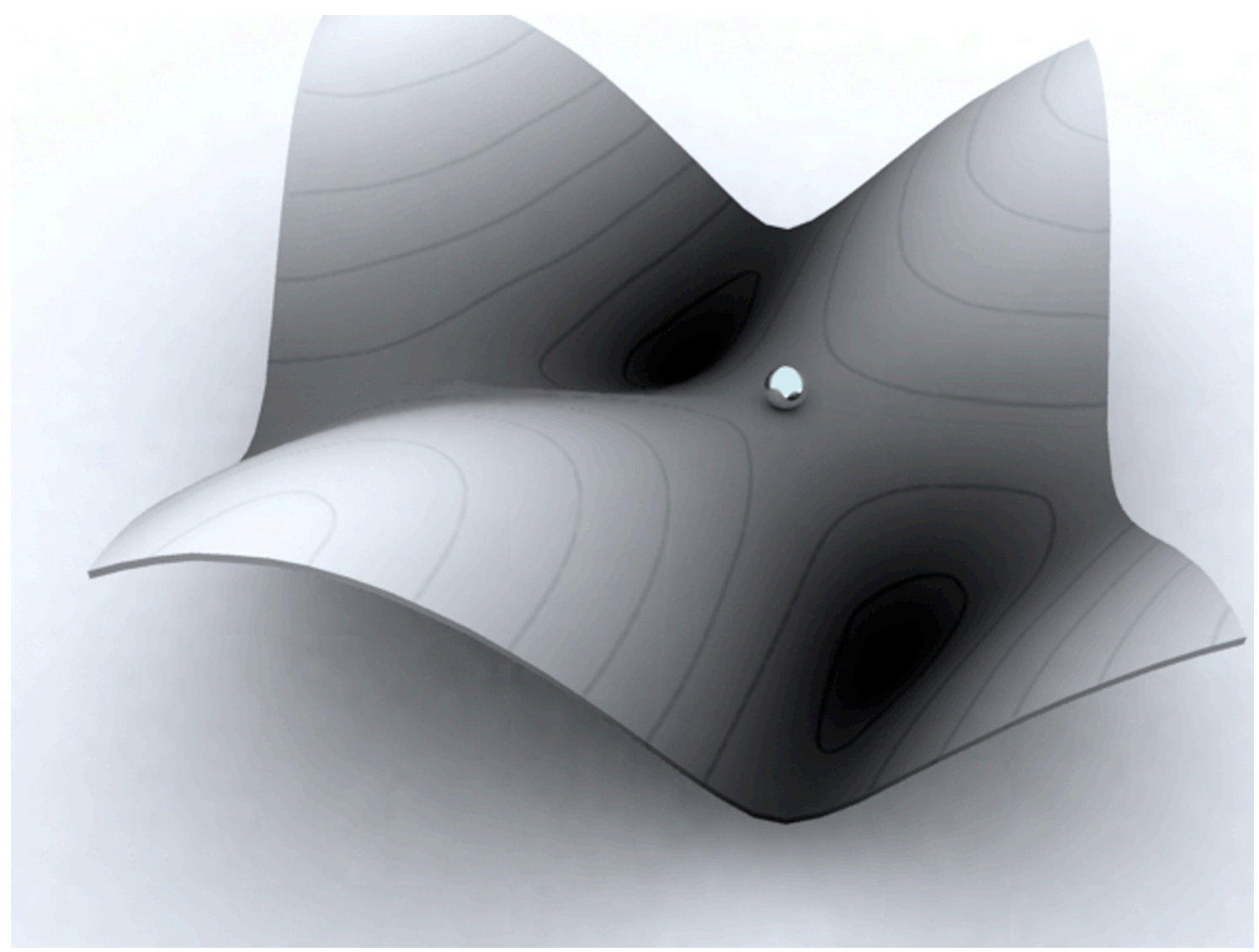

Figure 6: System portrait

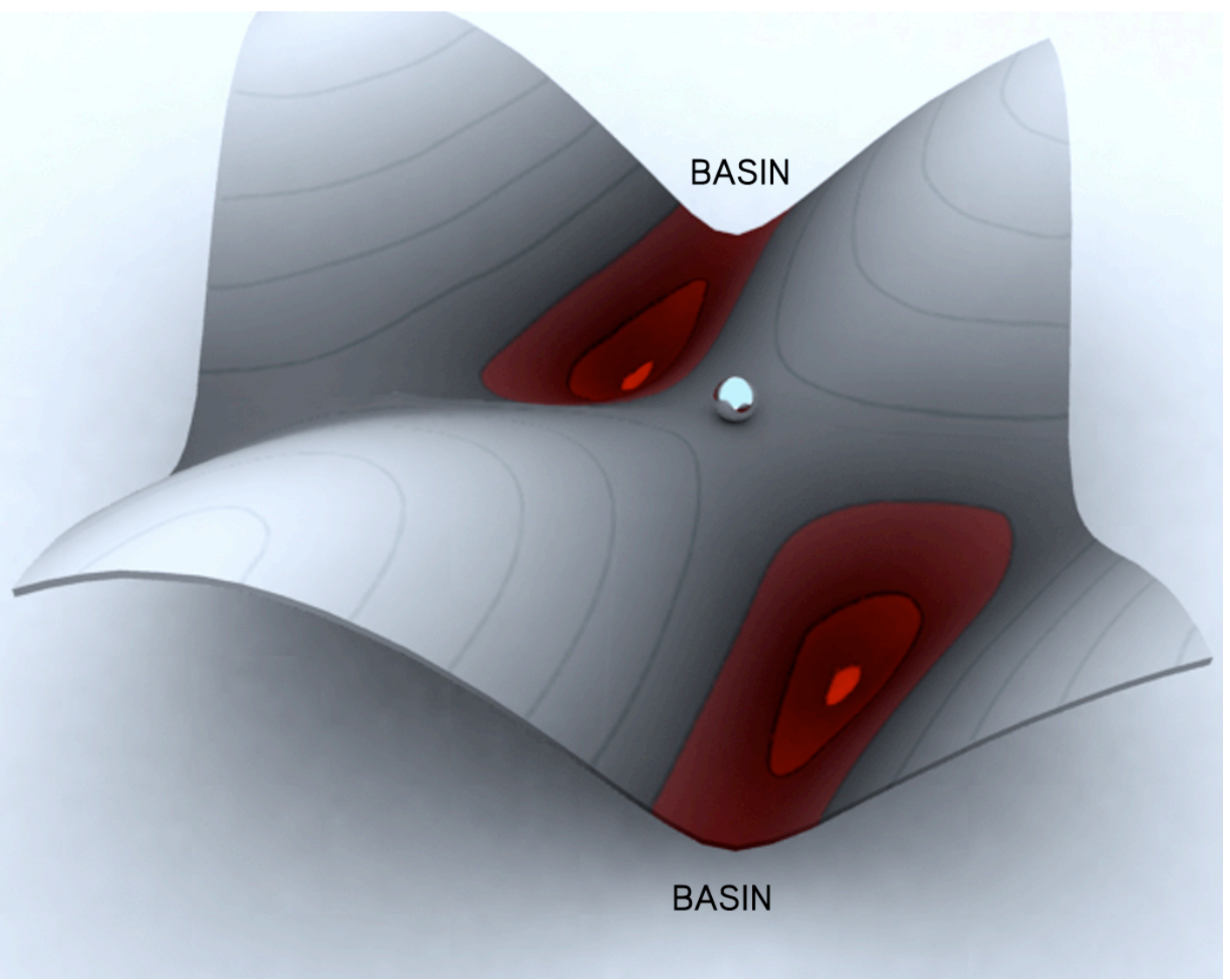

Figure 7: System basins 


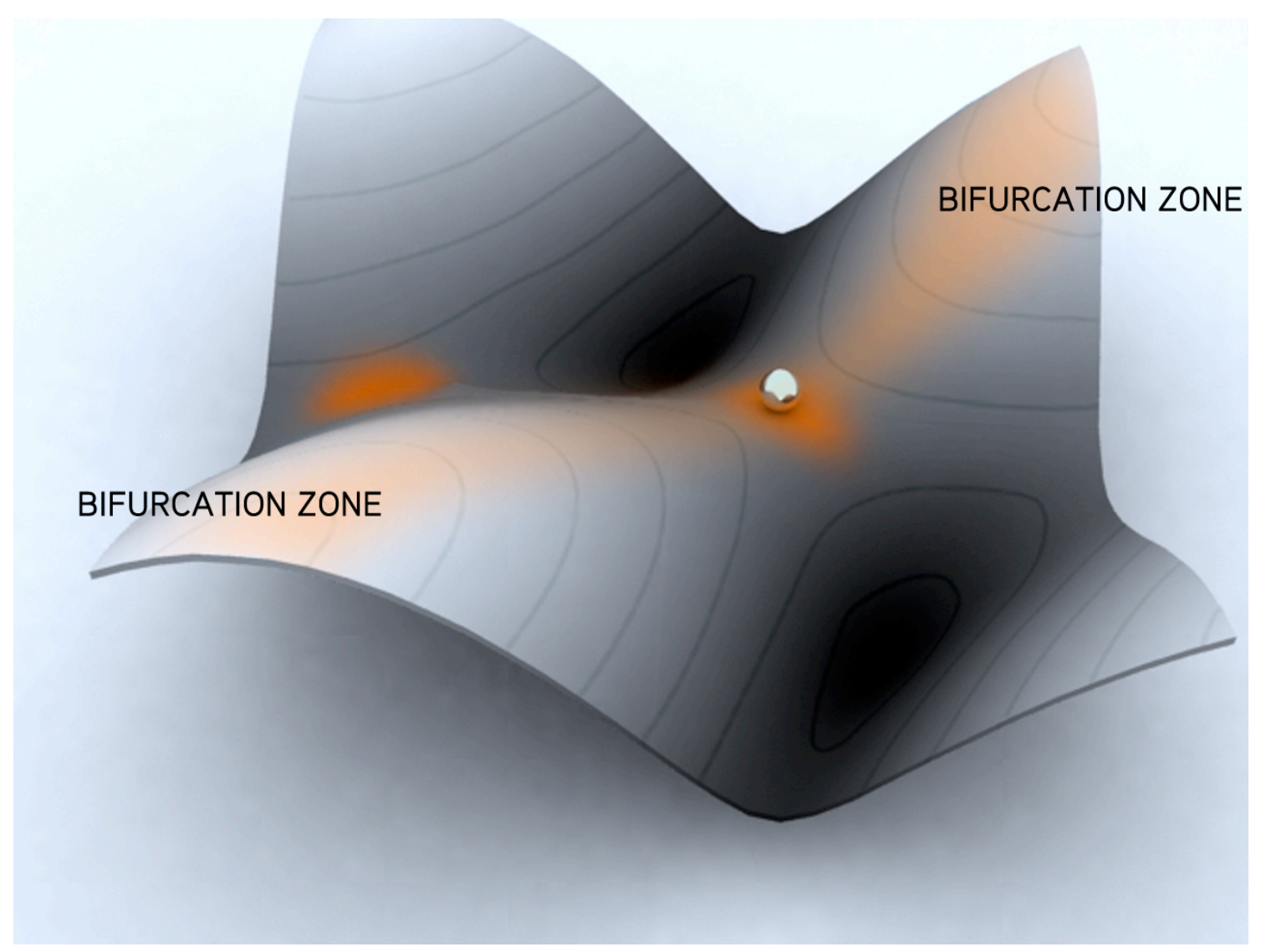

Figure 8: Bifurcation

Though the robot arm is useful as a pedagogical device to explain a search space, it is a highly simplified example. For more complex systems we will not be able to use a two dimensional graph. In fact every degree of freedom in a system will require its own axis; therefore for a system with $n$-degrees of freedom we will need an $n$-dimensional space to describe it.

Topological mathematics provides us with the resource to describe spaces of more than three dimensions. Topologically, a space can have an unlimited number of dimensions. The manifold is the mathematical 'description' of the state space; therefore the manifold describes all possible configurations of variables. A point located on the surface of that space will describe the particular configurations of each of the degrees of freedom of its component parts at any time, the movement of that point across this n-dimensional surface will describe the change in any of these potential configurations across time. This is essentially a meta-space that describes the systems potential. The specific behaviours of the system will emerge out of the constraints and tendencies of the topological space. It should be noted that there is some contention as to the status of these spaces with regards to the phenomena that they model. As Dyke notes:

"State spaces can be called "heuristics", "models", "epistemological devices" or what ever you like. But whatever we call them, we cannot be justified in imagining a real world "process" of the successive piling up of constraints in as necessarily being mirrored in our successive manipulations of a matrix. The notion of a state space is an analytical tool we use to design research." (Dyke 14)

Questions of fidelity or accuracy with regards to the phenomena being modelled are of course important in an analytical sense, but at no point can we say that there is an perfect equivalence between the phenomena and the model, only that certain salient characteristics of the phenomena have been incorporated into the modelling technique and that the these characteristics shape the rate and trajectory of an object as it moves through the model. As Delanda suggests

"(The) value of state space would be to reveal a topological isomorphism between singularities in the model and singularities in the physical system being modelled. " (Delanda 147) 


\section{Possibility}

This account of emergence as it has been described is still highly problematic for one very significant reason. If all the potential behaviours in a system are given at once as on the graph, and all that is left to occur is for them to be simply selected by a point in its trajectory through the model, then we are left with a system in which what is possible already pre-exists. Time would only operate to make real that which has always been a possibility located in space.

Gilles Deleuze provides a very precise way of understanding this space of potential that accords to 'time' its properly creative function and thus opens the opportunity for novelty or newness. He achieves this via a critique of the categories; 'possible' and 'real'.

"Every time we pose the question in terms of possible and real, we are forced to conceive of existence as a brute eruption, a pure act or leap which always occurs behind our backs and is subject to a law of all or nothing. What difference can there be between the existent and the non-existent if the non-existent is already possible, already included in the concept and having all the characteristics that the concept confers upon it as a possibility?

...to the extent that the possible is open to 'realisation', it is understood as an image of the real, while the real is supposed to resemble the possible. That is why it is so difficult to understand what existence adds to the concept when all it does is double like with like. Such is the defect of the possible: a defect which serves to condemn it as produced after the fact, as retroactively fabricated in the image of what it resembles. "(Deleuze.2:211)

For Deleuze a stable topology in which what is available to actualization already exists, is not worth having, since all it does is make real what previously existed as a possibility, thus doubling 'like with like'.

"That is to say, we give ourselves a real that is ready-made, preformed, pre-existant to itself, and that will pass into existence according to an order of successive limitations. Everything is already completely given: all of the real in the image, in the pseudo actuality of the possible." (Deleuze. 98)

\section{The virtual}

This state space or landscape of potential is what Deleuze would refer to as the virtual, with one important qualification that we will come to. The virtual is not physical and yet it is real, further not only is it real but it provides the generative space out of which physical form is developed. What is physical would be an actualisation of a particular location on this topological landscape.

As Bonta and Protevi have noted, the qualification that must be introduced, is that for Deleuze a properly virtual topology is unstable and dynamic (Bonta and Protevi 27). That is, the reservoir of potential forms out of which physical form emerges, is itself subject to variation. This is critical for Deleuze's ontology, because the stakes must not be limited to the variation in action that can take place within the limits of the system, what must be at stake is the very form of the virtual itself. A strongly emergent system is one in which this reservoir of potential is in variation with itself, so that what is available for development is itself subject to change. Weakly emergent systems would be systems in which the topology- that is the reservoir of potential forms on which actualisation processes can draw- is relatively stable and given only to local reconfigurations. This distinction between weak and strong emergence thus directly relates to type of spaces from which variation can potentially draw. It explains the limits of possible difference.

The point of understanding conceptually if not mathematically, all these features and their terminology, is that an emergent approach to design means that these specific articulations and characteristics of the virtual world become the levers and instruments, or better; the morphogenetic drives and structuring forces available to the designer. Understanding those singular zones of a systems topology- those areas of the virtual landscape in which behaviour is balanced between two different states- means that we know when and where to apply pressure to shift a systems behaviour. More importantly it promises an economy of effect since only a minimal effort is required to tip a system poised on the cusp of two different states. 


\section{Notes}

${ }^{1}$ It is important to note that that this mathematical space in which variations of the objects exist is not infinite in extension, it is bounded and has limits, rather we should say that it is infinite in density-a bounded infinity.

${ }^{2}$ What emerges with a new medium or practice more than a set of techniques or methodologies is a new series of problems in which existing techniques and concepts must be recast. Just as the 'problem' of authenticity only emerges with photography and mechanical reproduction, emergence carries with it its own series of lures and traps whose effect will be felt outside of the confines of computational or digital approaches to design.

\section{References}

Bach, Klaus and Frei Otto. IL 18. Forming Bubbles. Institute for Lightweight structures, University of Stuttgart, 1988.

Bergson, Henry. Matter and Memory. Paris: Presses Universitaires de France, 1959.

Bonta, Mark and John Protevi. Deleuze and Geophilosophy. Edinburgh: Edinburgh University Press, 2004.

Delanda, Manuel. Intensive Science Virtual Philosophy. London: Continuum, 2002.

Deleuze, Gilles. Bergsonism. Paris: Presses Universitaires de France, 1966. New York: Zone Books, 1998.

Deleuze, Gilles. Difference and Repetition. Paris: Presses Universitaires de France, 1968. New York: The Athlone Press Limited, 1994.

Dyke, Chuck. The Evolutionary Dynamics of Complex Systems. A Study in Biosocial Complexity. New York: Oxford university Press, 1988.

Elias, Hugo. Home page 10 July 2007 http://freespace.virgin.net/hugo.elias/models/m ik2.htm

Kauffman, Stuart A. "Antichaos and adaptation”. Scientific American 265.2 (1991): 78-84.

Krugman, Paul. The Self Organizing Economy. Cambridge: Blackwell Publishing, 1996.

Prigogine, llya. The End of Certainty. Paris: Editions Odile Jacob, 1996.

Silberstein, Michael and John McGeever. "The Search for Ontological Emergence." The Philosophical Quarterly 49.195 (1999): 182.

Strogatz, Steven H. Nonlinear Dynamics and Chaos. Cambridge: Perseus Books, 1994. 